\title{
Numerical simulation investigation of a new approach generating phase screens for modeling a turbulent media
}

\author{
She Chen, Hai-Xing Yan*, and Shu-Shan Li \\ (Institute of Mechanics, Chinese Academy of Sciences, Beijing 100080, China)
}

\begin{abstract}
In this paper, the theoretical base of the covariance approach for generating random phase screens is analyzed, and corresponding computer program is compiled. Preliminary numerical simulation investigation of this new approach is carried out. We propose to use three methods to evaluate the generated phase screens in a combining way. It is found that a comparison of the phase structure function of generated phase screens with the theoretical one is not enough and often inefficient. Open loop results and close loop results by using phase screens generated by the covariance approach are obtained and compared with those by using phase screens generated by the spectral approach for the first time within our knowledge. It is shown that the phase screens generated by covariance approach include more abundant frequency components than those generated by the spectral approach and these frequency components have obvious influences on the open loop results and the close loop results.
\end{abstract}

Keywords: Atmospheric propagation of optical wave, Atmospheric turbulence, Phase screen, Covariance, Numerical simulation, Phase compensation

\section{INTRUODUCTION}

Numerical simulation of an adaptive optics (AO) system for optical imaging and light propagation through a turbulent atmosphere requires an accurate simulation for atmospheric propagation of an optical wave. Multiple phase screen method is typically used to describe turbulent atmosphere. The structure constant of the index of refraction fluctuations, $C_{n}{ }^{2}$, is used to characterize the turbulence strength. ${ }^{[1]}$ The propagation path through the turbulent media can be divided into several segments which may have different lengths. It is thought that each segment may deform the phase of the optical wave independently. The contribution of the turbulent media segment to the phase of the optical wave can be "pressed" into a very thin phase screen and added to the initial phase of the wave. It is assumed that the phase screen does not have a significant influence on the amplitude of the wave. The amplitude of the optical wave is changed only in the propagation process of the wave with the deformed phase. It means that changes in phase and amplitude of an optical wave can be treated separately.

The phase screen generator is numerically investigated in this paper. The computer program used for creating random arrays of phase values on a grid of sample points is compiled. The arrays have the same statistics as the turbulenceinduced atmospheric phase. The statistics of phase required to create the desired phase screens have been presented in Ref. [1]. The spectral approach has generally been used to generate phase screens, which are not modeled well in low frequencies of the Kolmogorov or von Karman spectrum. A new approach used for simulating effects of atmospheric turbulence is named as the covariance approach. It is quite different from the conventional spectral

* Correspondence: Email: hxyan@imech.ac.cn; Phone: (86) 10 62554123; Fax: (86) 1062561284. 
approach. This approach can offer better performance in terms of giving the spatial and temporal statistics that match the desired statistics. Although this approach has several specific advantages, its application in a simulation of optical wave propagation through the turbulent atmosphere and $\mathrm{AO}$ system has not been studied, and its comparison with the spectral approach has not analyzed in details until now.

\section{PHASE SCREEN GENERATION APPORACH}

There are several approaches used to generate random phase screens with the proper point statistics and spatial and temporal correlation properties ${ }^{[2]}$. Conventional FFT based simulation or the spectral approach has been widely used to simulate atmospheric turbulence. ${ }^{[3,4]}$ In this approach, the very large spatial scales (which are responsible for low-order optical effects such as tilt) cannot be properly represented in a simulation that is limited by the FFT algorithm. In order to properly account for the low frequency contribution to the phase screens, it is necessary to sample the spectrum at scales outside of the inertial range ${ }^{[5-7]}$. A totally different approach i.e. the covariance approach was proposed several years ago. ${ }^{[1,2,8-10]}$ This approach does not have such low frequency limitation.

The Kolmogorov spectrum and the von Karman spectrum are commonly used in the atmospheric optics to describe a uniform isotropic turbulence. The Kolmogorov spectrum $\Phi_{n}^{K}(\kappa, z)$ is given by the following equation:

$$
\Phi_{n}^{K}(\stackrel{\mathrm{r}}{\kappa})=0.033 C_{n}^{2} \kappa^{-11 / 3}=0.033 C_{n}^{2}\left(\kappa_{x}^{2}+\kappa_{y}^{2}+\kappa_{z}^{2}\right)^{-11 / 6}
$$

A simplified form of the von Karman spectrum that ignores the inner scale effect is given by:

$$
\Phi_{n}^{V}=\frac{0.033 C_{n}^{2}(z)}{\left(\kappa^{2}+4 \pi^{2} / L_{0}^{2}\right)^{11 / 6}}
$$

where $L_{0}$ is the outer scale of the atmospheric turbulence.

When using the delta function basis set, theoretical formulae for calculating the element of covariance matrix of the phase fluctuations for an $N$-Layer atmosphere are presented by Ref. [1], wherein Eq. (3.205) is for the von Karman spectrum and Eq. (3.214) is for the Kolmogorov spectrum.

Using the relationship of structure function and the correlation function

$$
D_{\psi}(\stackrel{\mathrm{r}}{\rho})=2\left[\Gamma_{\psi}(0)-\Gamma_{\psi}(\stackrel{\mathrm{r}}{\rho})\right]
$$

we can obtain the phase structure function of von Karman power spectrum:

$$
\begin{aligned}
& D_{\phi}(\stackrel{\mathrm{r}}{\rho})=2 \times 3.089 \times \sum_{i=1}^{N} r_{o_{i}}^{-5 / 3}\left[0.189\left(\frac{L_{0}}{\pi}\right)^{5 / 3}-\frac{\left(L_{0} / 2 \pi\right)^{5 / 6} K_{5 / 6}\left[2 \pi \rho / L_{0}\right] \rho^{5 / 6}}{2^{5 / 6} \Gamma[11 / 6]}\right] \\
& =6.178 \times \sum_{i=1}^{N} r_{o_{i}}^{-5 / 3}\left[0.189\left(\frac{L_{0}}{\pi}\right)^{5 / 3}-\frac{\left(L_{0} / 2 \pi\right)^{5 / 6} K_{5 / 6}\left[2 \pi \rho / L_{0}\right] \rho^{5 / 6}}{2^{5 / 6} \Gamma[11 / 6]}\right]
\end{aligned}
$$

where $r_{o_{i}}$ is defined by

$$
r_{0_{i}}=0.185\left[4 \pi^{2} /\left(k^{2} C_{n_{i}}^{2} \Delta z_{i}\right)\right]^{3 / 5}
$$

For Kolmogorov power spectrum, the phase structure function is:

$$
D_{\psi_{i}}(\rho)=6.88\left(\rho / r_{o_{i}}\right)^{5 / 3} \text {. }
$$


Direct simulation of phase screens can be achieved by using Singular Value Decomponent (SVD). Using SVD, the covariance matrix $\Gamma_{\phi}$ can be factorized into a product of three square matrix, ${ }^{[1]}$ the eigenvalue diagonal matrix of $\Lambda$ which is a matrix with eigenvalues on the diagonal and zero elsewhere, eigenvector column of $U$, and its transposed column of $U^{T}$ where the superscript $T$ represents the matrix transpose operator:

$$
\Gamma_{\phi}=U \Lambda U^{T}
$$

We generate a vector $b$ of uncorrelated Gaussian random variables with zero mean and variance $\Lambda$ of length P by $\mathrm{Q}$.

$$
E\left(b b^{T}\right)=\Lambda
$$

Random phase screens are formed using:

$$
a=U b
$$

This vector $a$ is the column vector composed of the weights. Recall that when using the delta function basis set, the actual values of the weights correspond to the phase.

It is straightforward to verify this new vector $a$ has a covariance of $\Gamma_{\phi}$ by using Eqs. (9) and (8):

$$
E\left\{a a^{T}\right\}=E\left\{U b(U b)^{T}\right\}=E\left\{U b b^{T} U^{T}\right\}=U E\left\{b b^{T}\right\} U^{T}=U \Lambda U^{T}=\Gamma_{\phi}
$$

\section{GENERATING AND VERIFYING PHASE SCREENS}

In order to generate phase screens, the following steps can be carried out. First, based on Eq. (3.205) and Eq. (3.214) given by Ref. [1] and using widely available mathematical computation software packages, ${ }^{[11]}$ the covariance matrix $\Gamma_{\phi}$ can be computed. Second, the covariance matrix corresponding to a specific power spectrum can be factorized by using special SVD algorithm. Third, a vector $b$ of uncorrelated Gaussian random variables with zero mean and variance $\Lambda$ of length $\mathrm{P}$ by $\mathrm{Q}$ can be obtained by using a proper random number generator. Finally, phase screens can be generated by the above-mentioned matrix operation. Here, FORTRAN has been used to accomplish such computation.

After getting the generated phase screens, evaluation of these screens is an important issue. Three different methods are proposed to evaluate the generated phase screens in this paper.

Firstly, the accuracy of generated phase screens can be evaluated by means of a comparison of their phase structure function ${ }^{[1]}$ over an appropriately sized ensemble with the theoretical phase structure function described in Eqs. (4) and (6). Recall that Eqs. (4) and (6) are the phase structure function for the von Karman power spectrum and the phase structure function for the Kolmogorov power spectrum, respectively. It is shown later that the agreement in this comparison is necessary for an appropriate phase screen, but is neither enough nor efficient. That is to say, in many cases different generated phase screens show an excellent agreement between their phase structure functions and the theoretical one, but quite different results and/or behaviors can be obtained from them.

The second method is directly to compare the covariance matrix of the generated phase screens $\Gamma_{\% \circ}$ and the theoretical covariance matrix $\Gamma_{\phi}$. The covariance matrix of the simulated phase screens $\Gamma_{\not \%}$ can be calculated as follows

$$
\Gamma_{\phi^{\circ}}=E\left[\not \phi^{\circ} 0\right]
$$


where $\mathscr{\phi}^{\prime}$ is the vector of the generated random phases, $E[\bullet]$ is the ensemble average operator. The mean square error per covariance array element, $\varepsilon^{2}$, was computed, where $\varepsilon^{2}$ is given by:

$$
\varepsilon^{2}=\sum\left(\Gamma_{\phi}-\Gamma_{\phi \circ}\right)^{2} /(P \times Q)^{2}
$$

where the summation is taken over all points in the covariance matrices. For the calculation corresponding to the results above, we can compare the differences between the covariance matrices of different generated phase screens and the theoretical one.

The third method of evaluation is based on a simulation computation of the optical wave propagation through the multiple generated phase screens (the open-loop results) and the phase compensation of its deformed wavefront (the close-loop results) ${ }^{[4]}$. For example, the computational results of the open-loop and close-loop results of phase screens generated by the covariance approach can be compared with those of phase screens generated by the spectral approach. It is shown later that by using the simulation computation of the optical wave propagation through a turbulent media represented by the generated phase screens and its phase compensation, the difference between two approaches can be compared and investigated more thoroughly and more deeply.

It is shown in this paper that in order to evaluate the generated phase screens more definitely, these three methods must be combined.

\section{TWO PROBLEMS IN PHASE SCREEN GENERATION USING COVARIANCE APPROACH}

While using covariance approach to produce the random phase screens, it is found that decomponent of the covariance matrix and the choice of the Gaussian random number generator are two problems to need studying carefully.

\subsection{Decomponent of the covariance matrix}

After getting the covariance matrix, the next problem is how to decompose it. This step is to decompose the covariance matrix into a product of several matrices. In Ref. [1] and Refs. [6, 7], Cholesky factorization and SVD are used to decompose the covariance matrix, respectively.

We found by means of a numerical experiment that when using Cholesky factorization to decompose the covariance matrix, in many cases it is impossible to realize the decomponent. It is because the Cholesky factorization can be realized to factorize the covariance matrix into a product of two square matrices only when the covariance matrix is guaranteed to be real, symmetric, and positive definitely. The positive definite nature of the covariance matrix without including the time factor was proved in Ref. [12], but the positive definite nature of the covariance matrix after introducing the time factor was not proved.

In actual operation, the SVD algorithm ${ }^{[11]}$ is adopted to decompose the covariance matrix. Its requirement for the decomposed matrix is relatively low. It only requires the matrix is Hermitian, and the covariance matrix here obviously meets this requirement. In the following calculations including many different scenes, the SVD algorithm is used to decompose the covariance matrix without failing. We think that the SVD algorithm can be used to decompose the covariance matrix more widely than the Cholesky factorization does.

Certainly, in the viewpoint of the algorithm complexity, the computational amount of the SVD algorithm is much more than the Cholesky factorization. However, the SVD algorithm is needed to be carried out only once in the process of phase screen generation, further computations are to produce the new random number array and to carry out simple matrix operation again and again for generating new phase screen(s), so that the computational amount of initial stage is not a serious problem to the whole process of generating random phase screen(s). 


\subsection{Influence of the random number generator to the phase screen generation}

Another problem in the process generating random phase screens is the choice of the random number generator. It can be known from Eq. (8) that this is an important factor influencing the phase screens' performance. Different random number generators in Refs. [11, 13] have been compared in this section.

A random number generator for producing random numbers between 0 and 1 must be used in the Gaussian random number generator Gasdev in Ref. [11]. RAN and RAN0-RAN4 are different random number generators for producing random numbers between 0 and 1. Among them, RAN is the internal function of Compaq Visual FORTRAN6.6 system, RAN0 to RAN4 are the standard functions offered in Ref. [13], and DRNNOA and DRNNOR are Gaussian random number generators adopted in Ref. [11].

Mean value and variance of 1,000,000 random numbers between 0 and 1 produced by these random number generators are calculated, but for briefness the results are not shown here. It is shown that all the random number generators for producing random numbers between 0 and 1 are good enough, it is very difficult to find out the better one(s) only based on comparing the variance and mean value of the produced random numbers.
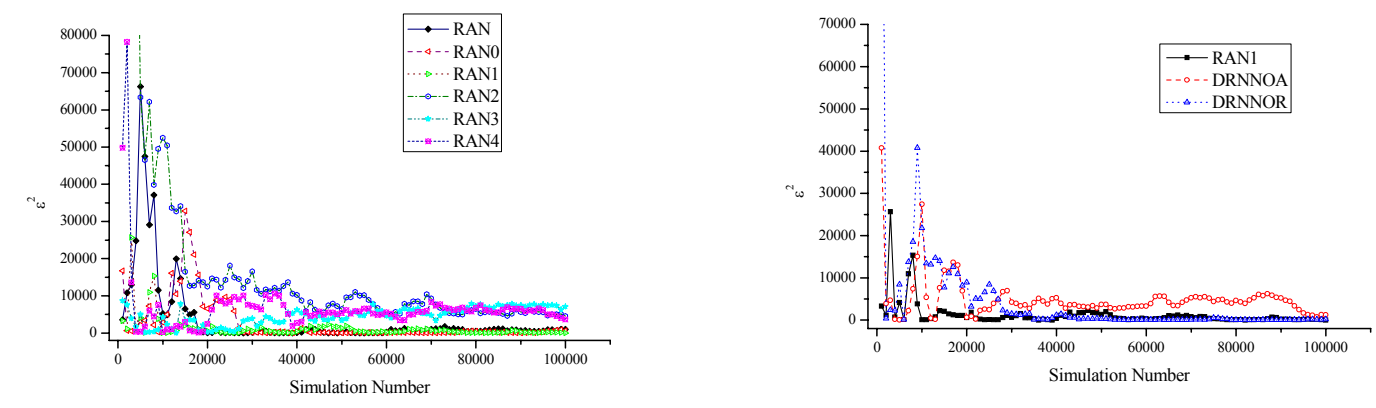

Figure 1 Test of different random number generators

In order to evaluate the influence of different standard random number generators on the phase screens generated by the covariance approach, we adopt a typical scene mentioned in Ref. [1] and produce 100,000 random phase screens, then calculate the average $\varepsilon^{2}$ using Eq. (12). The results are shown in Fig. 1. On the basis of $\varepsilon^{2}$, we can judge which random number generators are better for generating the phase screens by using the covariance approach. It is shown in Fig. 1 that average $\varepsilon^{2}$ of four generators, Gasdev ${ }^{[11]}$ adopted RAN, RAN0 and RAN1, and the DRNNOR ${ }^{[13]}$ can reach very low level for 100,000 times averaging, and the average $\varepsilon^{2}$ keep constant after 25,000 times averaging. In contrast, results of other generators are relatively poor. In view of above, any one of these four random number generators can be used to obtain better results. In following calculation, we have selected Gasdev as the Gaussian random number generator, and RAN1 has been used in Gasdev to produce the false random number between 0 and 1 .

On another hand, the phase structure functions of the random phase screens generated by using different random number generators have been compared with the theoretical formula; and all these generators show a quite similar behavior. These comparisons are not shown here for the sake of clarity. This indicates that it is not enough to judge the quality of the generated phase screens only based on comparing the phase structure function. In some cases, the average $\varepsilon^{2}$ is more sensitive to distinguish the slight changes in generated phase screens.

\section{EXAMPLES OF GENERATED PHASE SCREENS AND DISCUSSION}

In this section, some examples of phase screens generated by the covariance approach by using the delta function basics set are shown. These random phase screens are separated in time. In order to compare with the result of Ref. [1], 
the von Karman spectrum and the Kolmogorov spectrum are used with the SLC-Night $\mathrm{C}_{\mathrm{n}}^{2}$ profile shown in Ref. [1], and the same four layers model of this profile is used here. The value of the Fried parameter is $r_{0}=0.103 \mathrm{~m}$. The outer scale for von Karman spectrum is set at $L_{0}=100 \mathrm{~m}$. For the von Karman spectrum results, the length of one side of the square aperture is set equal to $1 \mathrm{~m}$, and there are 21 phase sample points across one side of the aperture (i.e., $\mathrm{P}=21 \times 21$ ), which provided a sample spacing of $\Delta x=5 \mathrm{~cm}$. The Kolmogorov spectrum results are calculated using Gegenbauer polynomial technique for a $1 \mathrm{~m}$ radius aperture. The Kolmogorov spectrum results are for the $1 \mathrm{~m}$ by $1 \mathrm{~m}$ square centered within the $1 \mathrm{~m}$ radius circle. Results for three temporal realization of the phase screen (i.e., $\mathrm{Q}=3$ ) are shown, which require computation and factorization of $1323 \times 1323$ element covariance arrays.

Figure. 2 shows the surface plots and grey scale images of three random time-sequential phase screens created using the von Karman spectrum. The grey scale plots were created by linearly stretching the phase screen values between 0 and 255 gray levels. The wind velocities for each of the four layers are identical, with $v_{x}=0 \mathrm{~m} / \mathrm{s}$ and $v_{y}=10 \mathrm{~m} / \mathrm{s}$, where $v_{x}$ and $v_{y}$ are the $x$ - and $y$-directed component of $\hat{v}(z)$. It is easy to note the movement of the major structures in phase screen is $y$-direction, because the time between phase screens is set to be $0.01 \mathrm{~s}$, the movement of the major structure is exactly two sample spacings of $10 \mathrm{~cm}$ in the $y$ direction. From the grey scale plot, the movement is along the downward direction (corresponding to the $y$ increasing direction). However, it is very easy to notice that certain deformation of the phase screen surface has taken place in addition to the major structure' movement.

Phase screens for the Kolmogorov spectrum are also obtained by using the same random number seed used to generate Fig. 2. The data and figure show that the phase screens are quite similar although different power spectrums are adopted. In the set of phase screens using Kolmogorov spectrum, there are the movement of major structure and the deformation of phase screen surface as well. For briefness, these phase screens are not presented here.

These results are very well comparable to those of Ref. [1].

Effects of the lateral wind and the Taylor's frozen field hypothesis in the spectral approach are expressed in the following way: to produce large enough phase screens using the spectral approach, to choose an area among them as the initial area corresponding to the optical path of beacon. Because of the lateral wind, phase screens move certain distances within the time interval along the wind direction, which is perpendicular to the light propagation direction. Because the large phase screens remain to be constant in the whole process, there is no deformation of surfaces of phase screens.

Like this, an important problem appears: based on the same Taylor's frozen field hypothesis, movement of major structure and surface deformation exist in phase screens generated by the covariance approach, however, there is no surface deformation in addition to the movement of major structure in phase screens generated by using the spectral approach; which approach is better for generating phase screen(s)?

Taylor's frozen field hypothesis is utilized in the spectral approach after the generation of the phase screens. This is why the phase screens only move along the wind direction, and have no deformation at all. On the other hand, in the covariance approach the frozen field hypothesis is introduced in the process of calculating the covariance matrix, and the effect of such hypothesis has been "naturally" included in the generated phase screens. By investigating the algorithm, it is found that the number of singular value in the eigenvalue diagonal matrix is more than the product $P$ of the grid point numbers in the $x$ and $y$ dimension of phase screen. Generally, a singular value corresponds to space frequency component(s). More none-zero singular value appeared in the eigenvalue diagonal matrix means that there are more space frequency component(s) to be included in the phase screens generated by the covariance approach. The influence of these more abundant frequency component(s) is to produce the phase screen surface deformation. The real atmospheric turbulence should be much more complicated than the Taylor's frozen field hypothesis. At sequential 
moments, in addition to the effect of the lateral wind, real-time deformations exist in the atmospheric turbulence, and frequency components should be much more abundant than what a limited density of phase screen can reflect. The timesequential phase screens generated by using the covariance approach are considered as a whole at the beginning, and

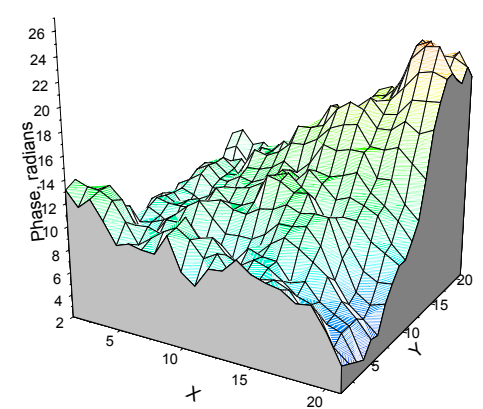

(a)

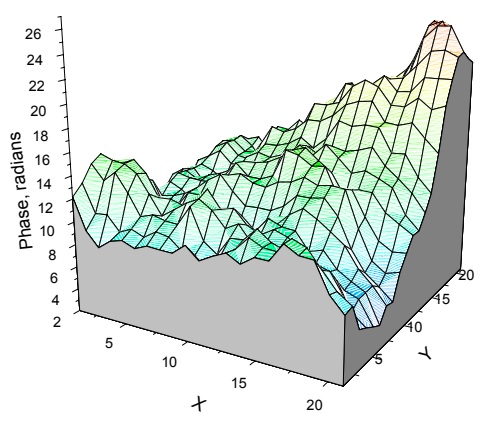

(c)

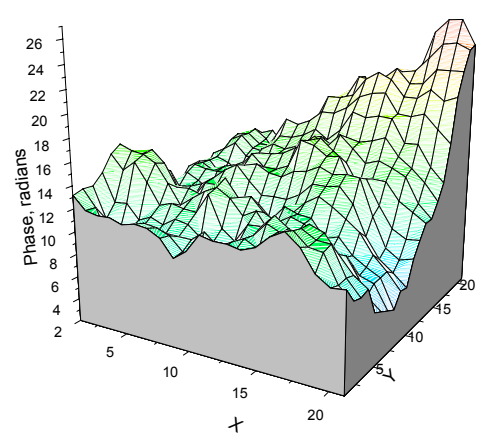

(e)

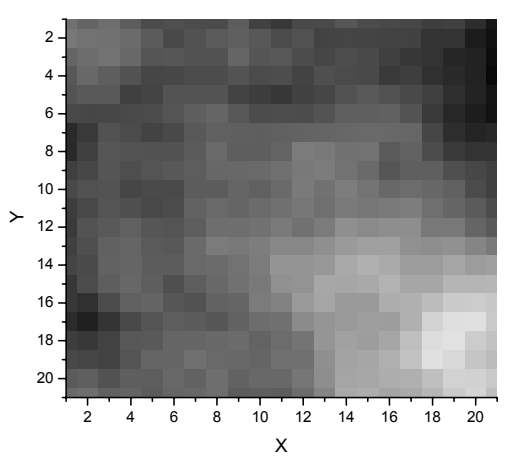

(b)

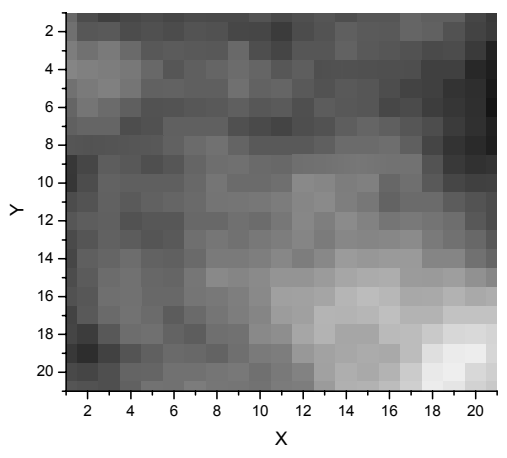

(d)

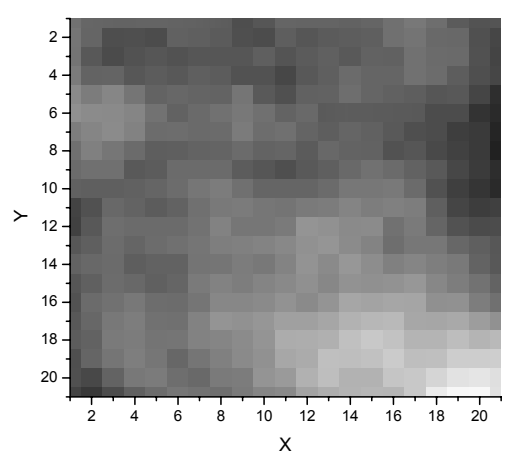

(f)

Figure 2 Three random, time sequential images for the case of atmospheric layers moving in the same direction and speed, and a von Karman spectrum: (a) surface plot of phase screen, $t=0 \mathrm{~s}$; (b) grey scale image of (a); (c) surface plot of phase screen, $t=0.01 \mathrm{~s} ;$ (d) grey scale image of (c); (e) surface plot of phase screen, $t=0.02 \mathrm{~s} ;$ (f) grey scale image of (e). 
then they are cut into the time-sequential portions having limited size. Thus, more information than those expressing the major structure shift are included in these phase screens. From this point of view, the phase screens generated by the covariance approach should be more realistic than those generated by the spectral approach.

In order to verify above-mentioned thought, some singular values having smaller amount have been deleted from the singular value matrix when the number is greater than space sampling points. This is equivalent to removing the effect of the more abundant space frequency components in the covariance approach. After such artificial treatment, the movement of major structure remains in the generated phase screens, but the deformation of phase screen surface disappears. That is to say, the generated phase screens after this treatment are identical to those generated by the spectral approach. In fact, careful checking and comparing the data in the time-sequential phase screens shows that phase data in each previous phase screen after shifting two sample spacing in the $y$ direction are exactly same to

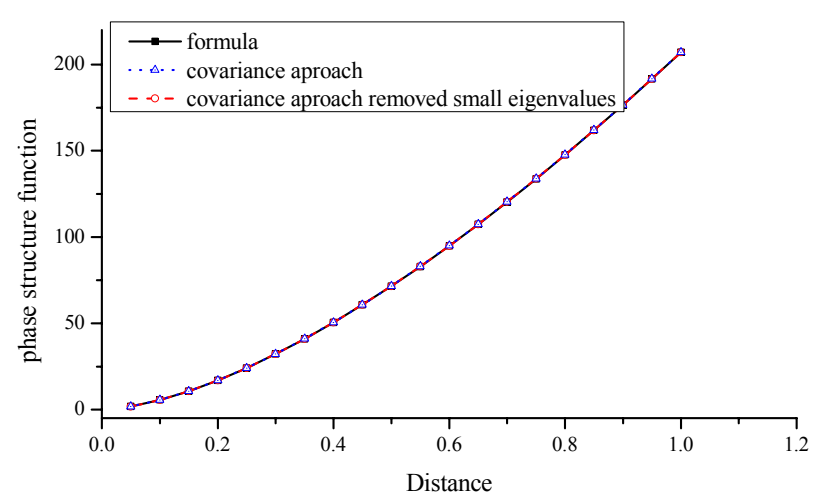

Fig. 3 Comparison of phase structure functions

those in the successive phase screen after removing "extra" singular values. It is shown that more abundant frequency components exist in the phase screens generated by the covariance approach and these components produce the surface deformation in the generated phase screens.

For checking the influence of this treatment on the phase structure function of the generated phase screens, abovementioned 100,000 times average phase structure functions before and after removing smaller eigenvalues are calculated and compared with the theoretical one. Fig. 3 shows the corresponding result. Fig. 3 shows that this treatment does not have obvious influence on the phase structure function, the phase structure functions before and after the treatment are very well comparable to the theoretical one. It means that the effluences of the more abundant space frequency components cannot be reflected in the phase structure function. It is shown again that it is not enough only to compare the phase structure function of the generated phase screen(s) with the theoretical one for verifying quality of the generated phase screen(s).

\section{RESULTS OF LIGHT PROPAGATION AND PHASE COMPENSATION AND DISCUSSION}

In this section, simulation computational results of atmospheric propagation of a optical wave and its phase compensation using random phase screens generated by the covariance approach and the spectral approach are presented and compared. This is one of the important methods to evaluate the covariance approach as well.

Six scenes are chosen as the simulation scenes. Similar to our previous work, ${ }^{[4]}$ long-exposure results of atmospheric propagation without and with static phase compensation having time delay are calculated using 3000 sets of random phase screens generated by two approaches. In our previous paper, ${ }^{[4]}$ phase screens generated by the spectral approach without and with low frequency correction are used to do the simulation computation separately. The results of von Karman (VON) spectrum and Kolmogorov (KOL) spectrum are calculated separately, using Strehl ratios, STRC and STRCC,$^{[4]}$ as the evaluation parameter. The main computational conditions are as follows: $3000 \mathrm{~m}$ horizontal propagation path is chosen; the whole propagation path is evenly separated into 10 phase screens; the focusing light beam is adopted; the distances between sampling points of successive phase screens are $4.04 \mathrm{~cm}, 3.62 \mathrm{~cm}, 3.21 \mathrm{~cm}, 2.80 \mathrm{~cm}, 2.38 \mathrm{~cm}, 1.97$ $\mathrm{cm}, 1.56 \mathrm{~cm}, 1.15 \mathrm{~cm}, 0.73 \mathrm{~cm}, 0.32 \mathrm{~cm}$, respectively; a constant lateral wind speed along $x$ direction is used for 10 
phase screens, the wind speed of y direction is chosen as $0 ; C_{n}{ }^{2}$ is assumed to be uniform along the optical path; the grid number of calculation is $32 \times 32$; the delay time is $2.75 \mathrm{~ms}$; the light wavelength is $632.8 \mathrm{~nm}$; the aperture size is $60 \mathrm{~cm}$.

Table 1 Comparison of simulation results of atmospheric propagation and phase compensation using phase screens generated

by the covariance approach and the spectral approach (I)

\begin{tabular}{|c|c|c|c|c|c|}
\hline \multirow{2}{*}{\multicolumn{2}{|c|}{ Computational condition }} & \multicolumn{2}{|c|}{ Open loop } & \multicolumn{2}{|c|}{ Close loop } \\
\hline & & STRC & STRCC & STRC & STRCC \\
\hline \multirow{4}{*}{$\begin{array}{c}\text { Wind velocity } \\
\begin{array}{c}2.22 \mathrm{~m} / \mathrm{s} \\
r_{0}=9.504 \mathrm{~cm}\end{array}\end{array}$} & Spectral approach without low frequency correction & 0.1197 & 0.4411 & 0.9684 & 0.9757 \\
\hline & Spectral approach with low frequency correction & 0.09879 & 0.3466 & 0.9677 & 0.9736 \\
\hline & Covariance approach for VON & 0.02465 & 0.1035 & 0.9206 & 0.9467 \\
\hline & Covariance approach for KOL & 0.01885 & 0.07922 & 0.9299 & 0.9502 \\
\hline \multirow{4}{*}{$\begin{array}{c}\text { Wind velocity } \\
2.42 \mathrm{~m} / \mathrm{s} \\
r_{0}=3.051 \mathrm{~cm}\end{array}$} & Spectral approach without low frequency correction & 0.01930 & 0.07356 & 0.8280 & 0.8693 \\
\hline & Spectral approach with low frequency correction & 0.01385 & 0.05795 & 0.8206 & 0.8676 \\
\hline & Covariance approach for VON & 0.008950 & 0.03875 & 0.5522 & 0.6775 \\
\hline & Covariance approach for KOL & 0.008999 & 0.03897 & 0.5837 & 0.6853 \\
\hline \multirow{4}{*}{$\begin{array}{c}\text { Wind velocity } \\
\begin{array}{c}3.14 \mathrm{~m} / \mathrm{s} \\
r_{0}=1.716 \mathrm{~cm}\end{array}\end{array}$} & Spectral approach without low frequency correction & 0.008964 & 0.03902 & 0.4822 & 0.6074 \\
\hline & Spectral approach with low frequency correction & 0.008736 & 0.03755 & 0.4791 & 0.6158 \\
\hline & Covariance approach for VON & 0.009216 & 0.03983 & 0.08877 & 0.2356 \\
\hline & Covariance approach for KOL & 0.009244 & 0.03988 & 0.1042 & 0.2663 \\
\hline
\end{tabular}

Because of the limitation of computer capability, a larger grid number cannot be used and it is impossible to carry out a simulation of a practical AO system under the present condition. This is why the complete phase compensation ${ }^{[4]}$ is used in this paper. The complete phase compensation means that after a light beam passes through the turbulent atmosphere (i.e. the turbulence phase screens), the distorted wavefront can be obtained at the incoming aperture and an minus sign (-) is taken for all the grid point of the distorted wavefront in the emission aperture. Finally the phase compensated beam (the light beam having minus distorted wavefront) propagates in the opposite direction and through a translated (because of the time delay and the lateral wind) turbulent media again to reach the target. The complete phase compensation is much simpler than a practical AO system, but it represents a specific phase compensation of the distorted wavefront. Open loop results without phase compensation and close loop results with phase compensation are calculated. For briefness, results of only three scenes are shown in Table 1.

It is shown in Table 1 that difference between open loop results of two approaches is relatively apparent in the case of weaker turbulence. As the turbulence intensity gets stronger, the difference becomes smaller. In the case of strong turbulence, results of the covariance approach can be well compared with those of the spectral approach. In contrast, close loop results of two approaches can be well compared in case of weaker turbulence; as the turbulence gets stronger, close loop results of the covariance approach is obviously lower than those of the spectral approach. In addition, change of open loop results of the spectral approach after including the low frequency correction is relatively obvious in the case of weaker turbulence; it is not very obvious in the case of stronger turbulence; and change of close loop results of the spectral approach after including the low frequency correction is not very obvious in the case of either weaker turbulence or stronger turbulence. Further, difference of the covariance approach results using two different power spectrums, i.e. von Karman spectrum and Kolmogorov spectrum is quite small. In the case of weaker turbulence, the complete phase compensation works very well, but in the case of stronger turbulence phase compensation results become very poor. This regulation is true for both the spectral approach and the covariance approach.

Further, average phase structure functions of 3000 realizations of phase screens generated by the covariance approach using von Karman spectrum in the case of three different turbulence intensities have been calculated and shown in Fig. 4. The phase structure function of phase screens generated by the covariance approach using Kolmogorov 
spectrum, the theoretical one and the phase structure functions of phase screens generated by the spectral approach using von Karman spectrum with and without low frequency correction are shown in Fig. 4 also.

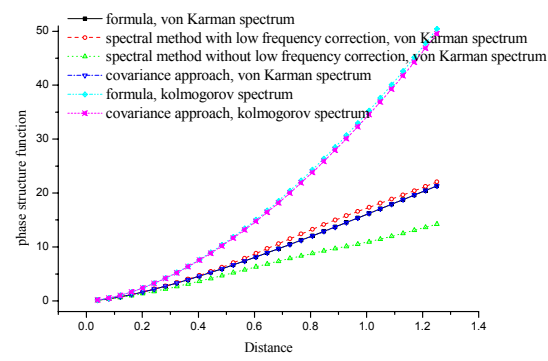

A

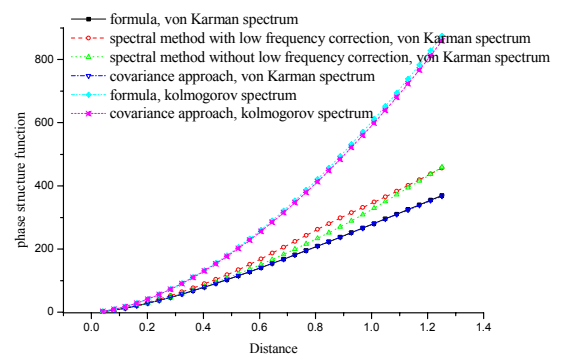

C

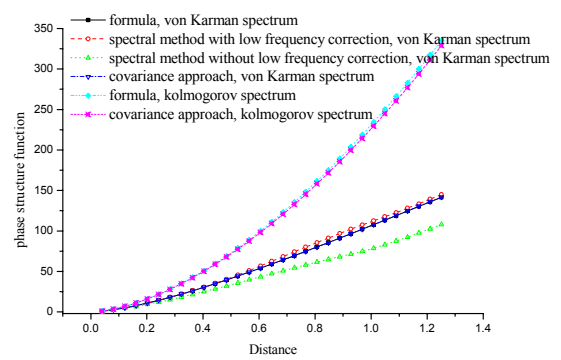

B

Fig. 4 Comparison of phase structure function

Computational condition: The first phase screen has

been selected from all ten screens, 3,000

realizations.

Fig. A Wind velocity $=2.22 \mathrm{~m} / \mathrm{s}, r_{0}=9.504 \mathrm{~cm}$,

Fig. B Wind velocity $=2.42 \mathrm{~m} / \mathrm{s}, r_{0}=3.051 \mathrm{~cm}$,

Fig. C Wind velocity $=3.14 \mathrm{~m} / \mathrm{s}, r_{0}=1.716 \mathrm{~cm}$.

In comparison to the theoretical phase structure function, results of the covariance approach are the best; the differences are very small in case of 3000 realizations. Results of the spectral approach can roughly compare to the theoretical result; without low frequency correction, a greater difference appears in the low space frequency region; after including the low frequency correction, the result of the spectral approach can quite well compare to the theoretical one; but the agreement is not as perfect as that of the covariance approach. It is noted that there is very great difference between the phase structure function produced by the von Karman spectrum and that of the Kolmogorov spectrum.

Open loop results and close loop results by using phase screens generated by the covariance approach for von Karman spectrum after removing "extra" smaller singular values are calculated and shown in Table 2. It is shown that after removing "extra" smaller eigenvalues artificially, the open loop results does not show great changes and the close loop results of the covariance approach tend to those of the spectral approach, although there still exist some obvious differences between them. This tendency is consistent with our expectation. After removing the frequency components corresponding to smaller eigenvalues, the correlation of successive phase screens has been improved and the phase surface deformation has been removed. This is why the close loop results of the covariance approach tend to those of the spectral approach and this tendency is more obvious for stronger turbulence. On the other hand, existence of some obvious difference between the covariance approach results and the spectral approach results indicates that the phase screens generated by the covariance approach are still not equivalent to those generated by the spectral approach even after removing "extra" smaller eigenvalues artificially in the covariance approach.

Following the similar thought, for further investigating the covariance approach, 4 largest eigenvalues and other "extra" smaller eigenvalues are removed from the eigenvalue matrix simultaneously. Then, open loop results and close loop results by using phase screens generated by the covariance approach for von Karman spectrum are calculated and shown in Table 3. It is shown that after removing 4 largest eigenvalues and other "extra" smaller eigenvalues artificially, the open loop results of the covariance approach show larger changes in the case of weaker turbulence and tend to those 
of the spectral approach; however, the open loop results of the covariance approach do not show obvious changes in the case of stronger turbulence; meanwhile, the close loop results of the covariance approach show some increases and tend to those of the spectral approach. After comparing to Table 2, it is found that these increases of the close loop results basically come from the treatment removing smaller eigenvalues. The treatment removing 4 largest eigenvalues does not have obvious influence on the close loop results (see Table 3).

Table 2 Comparison of simulation results of atmospheric propagation and phase compensation using phase screens generated by the covariance approach and the spectral approach (II)

\begin{tabular}{|c|c|c|c|c|c|}
\hline \multicolumn{2}{c}{ Computational condition } & \multicolumn{2}{c|}{ Open loop } & \multicolumn{2}{c|}{ Close loop } \\
\hline $\begin{array}{c}\text { Wind velocity } \\
2.22 \mathrm{~m} / \mathrm{s} \\
R_{0}=9.504 \mathrm{~cm}\end{array}$ & Covariance method - original & 0.02465 & 0.1035 & 0.9206 & 0.9467 \\
\cline { 2 - 7 } & Covariance method - smaller eigenvalues removed & 0.02623 & 0.1074 & 0.9394 & 0.9657 \\
\hline $\begin{array}{c}\text { Wind velocity } \\
2.42 \mathrm{~m} / \mathrm{s} \\
R_{0}=3.051 \mathrm{~cm}\end{array}$ & Covariance method - smaller eigenvalues removed & 0.009062 & 0.03925 & 0.6416 & 0.7882 \\
\hline $\begin{array}{c}\text { Wind velocity } \\
3.14 \mathrm{~m} / \mathrm{s} \\
R_{0}=1.716 \mathrm{~cm}\end{array}$ & Covariance method - smaller eigenvalues removed & 0.008942 & 0.03963 & 0.1823 & 0.3888 \\
\hline
\end{tabular}

Table 3 Comparison of simulation results of atmospheric propagation and phase compensation using phase screens generated

by the covariance approach and the spectral approach (III)

\begin{tabular}{|c|c|c|c|c|c|}
\hline \multicolumn{2}{|c|}{ Computational condition } & \multicolumn{2}{c|}{ Open loop } & \multicolumn{2}{c|}{ Close loop } \\
\hline $\begin{array}{c}\text { Wind velocity } \\
2.22 \mathrm{~m} / \mathrm{s} \\
R_{0}=9.504 \mathrm{~cm}\end{array}$ & $\begin{array}{c}\text { Covariance method - 4 largest and other } \\
\text { "extra" smaller eigenvalues removed }\end{array}$ & 0.06574 & 0.2408 & 0.9433 & 0.9659 \\
\hline $\begin{array}{c}\text { Wind velocity } \\
2.42 \mathrm{~m} / \mathrm{s} \\
R_{0}=3.051 \mathrm{~cm}\end{array}$ & $\begin{array}{c}\text { Covariance method - 4 largest and other } \\
\text { "extra" smaller eigenvalues removed }\end{array}$ & 0.01077 & 0.04580 & 0.6633 & 0.7906 \\
\hline $\begin{array}{c}\text { Wind velocity } \\
3.14 \mathrm{~m} / \mathrm{s} \\
R_{0}=1.716 \mathrm{~cm}\end{array}$ & $\begin{array}{c}\text { Covariance method - 4 largest and other } \\
\text { "extra" smaller eigenvalues removed }\end{array}$ & 0.009445 & 0.03990 & 0.1622 & 0.3886 \\
\hline
\end{tabular}

\section{CONCLUSION}

The covariance approach using delta function as base function is used to generate phase screens for the von Karman spectrum and the Kolmogorov spectrum and a preliminary numerical simulation investigation is carried out. Phase structure functions of phase screens generated by the covariance approach agree excellently with the theoretical one. The generated phase screens under the same conditions can be very well compared with those in Ref. [1]. It is found that the covariance matrix can not be factorized by using Cholesky factorization adopted in Ref. [1] in some cases; and the SVD algorithm can be adopted to realize the matrix factorization without failing. It is shown that different random number generators can influence on the generated random phase screens. Four better generators have been selected as the generator for generating the random numbers between 0 and 1 . We propose to use three methods to evaluate the generated phase screens in a combining way. It is found that a comparison of the phase structure function of generated phase screens with the theoretical one is not enough and often inefficient. By contrast, a comparison of open loop and 
close loop results by using generated phase screens can give a deeper insight. When the successive phase screens with temporal correlation property are generated, phase surface deformations have been found in addition to the movement of major structure. It is shown that these deformations can be removed by deleting the "extra" smaller eigenvalues in the covariance matrix and at the same time the phase structure function of generated phase screens does not have an obvious change. Open loop results and close loop results by using phase screens generated by the covariance approach are obtained and compared with those by using phase screens generated by the spectral approach for the first time within our knowledge. It is found that the open loop results of the covariance approach are lower than those of the spectral approach in case of weaker turbulence and its close loop results is obviously lower than those of the spectral approach in case of stronger turbulence. After deleting the "extra" smaller eigenvalues, the close loop results of the covariance approach tend to those of the spectral approach, and there is no obvious effect on the open loop results. If deleting 4 largest eigenvalues at the same time, the open loop results of the covariance approach tend to those of the spectral approach also. These indicate that the phase screens generated by the covariance approach include more abundant frequency components than the spectral approach and these frequency components have obvious influences on the open loop results and the close loop results.

This paper presents a preliminary numerical investigation of the covariance approach. There are a lot of important problems left for further work.

\section{REFERENCE}

1. M. C. Roggemann, B. M. Welsh, "Imaging through Atmospheres," CRC Press, 1996

2. T. Goldring, L. Carlson, "Analysis and implementation of non-kolmogorov phase screens appropriate to structured environments,” Proc. SPIE, vol.1060 243-264, 1989

3. J. A. Fleck, Jr., J. R. Morris, M. D. Feit, "Time-dependent propagation of high energy laser beams through the atmosphere,” Appl. Phys., vol. 10, 129-160, 1976

4. Hai-Xing Yan, Shu-Shan Li, De-Liang Zhang, She Chen "Numerical simulation of an adaptive optics system with laser propagation in the atmosphere," Appl. Opt., vol. 39, 3023-3031, 2000

5. J. M. Martin, S. M. Flatté, "Intensity images and statistics from numerical simulation of wave propagation in 3-D random media," Appl. Opt., vol. 27, 2111-2126, 1988

6. R. Flehlich, "Simulation of laser propagation in turbulent atmosphere," J. Opt. Soc. Am. A, vol. 39, 393-397, 2000

7. B.J. Herman, L.A. Strugala, "Method for inclusion of low-frequency contributions in numerical representation of atmospheric turbulence," Proc. SPIE. vol. 1221, 183-192, 1990

8. A.D. McAulay, "Artificial turbulence generation alternatives for use in computer and laboratory experiments," Proc. SPIE, Vol. 4493, 141-149, 2001

9. A. D. McAulay, "Generating Kolmogorov phase screens for modeling optical turbulence," Proc. SPIE, Vol. 4034, $50-57,2000$

10. C.M. Harding, R.A. Johnston, R.G. Lane, "Fast simulation of a Kolmogorov phase screen,” Applied Optics Vol. 38, 2161-2170, 1999

11. "Math library/Special function," in IMSL FORTRAN Subroutines for mathematical Applications, 1998

12. J. W. Goodman, "Statistical Optics," New York: John Wiley \& Sons, 1985

13. W. H. Press, B. P. Flannery, S. A. Teukolsky, W. T. Vetterling, "Numerical Recipes -The Art of Scientific Computing," Cambridge, UK: Cambridge University Press, 2 Edition, 1992) 